\title{
HYDROMAGNETIC RAREFIED FLUID FLOW OVER A WEDGE IN THE PRESENCE OF SURFACE SLIP AND THERMAL RADIATION
}

\author{
K. DAS \\ Department of Mathematics, A.B.N. Seal College, Cooch Behar \\ West Bengal, Pin:736101, INDIA \\ E-mail:kd_kgec@rediffmail.com \\ R.P. SHARMA \\ Department of Mathematics, JECRC University \\ Jaipur-303905, Rajasthan, INDIA \\ E-mail: ramprakash0808@gmail.com \\ P.R. DUARI \\ Dept. of Mathematics, Asansol Engineering College \\ Asansol, Pin-713305, INDIA \\ E-mail: pinakiranjanduari@gmail.com
}

\begin{abstract}
An analysis is presented to investigate the effects of thermal radiation on a convective slip flow of an electrically conducting slightly rarefied fluid, having temperature dependent fluid properties, over a wedge with a thermal jump at the surface of the boundary in the presence of a transverse magnetic field. The reduced equations are solved numerically using the finite difference code that implements the 3-stage Lobatto IIIa formula for the partitioned Runge-Kutta method. Numerical results for the dimensionless velocity and temperature as well as for the skin friction coefficient and the Nusselt number are presented through graphs and tables for pertinent parameters to show interesting aspects of the solution.
\end{abstract}

Key words: convective flow, heat transfer, surface slip, magnetic field, thermal radiation.

2010 Mathematics Subject Classification. 76W05, 76V05, 76P99

\section{Introduction}

Fluid flow over wedge shaped bodies is a phenomenon of great interest from both theoretical and practical point of view because of its considerable applications in mechanical, chemical, civil engineering and geophysical fields. Flow of a viscous incompressible fluid over a wedge was first studied by Falkner and Skan [1]. Rajagopal et al. [2] extended the problem of Falkner and Skan by considering the boundary layer flow of a second grade fluid over a wedge. A magnetohydrodynamics boundary layer flow of an electrically conducting fluid over a wedge surface has been discussed by many investigators [3-6], due to considerable theoretical and practical interest. The fluid behavior may be modelled as a rarefied gas [7]. The fluid is also treated as a rarefied gas, for large-scale problems with low density, for example, in outer space applications. A partial slip may occur on a stationary and moving boundary when the fluid is particulate such as suspensions, emulsions and polymer solutions. Thus considering an MHD liquid slip flow has a promising potential in numerous practical applications such as MHD micro pumps which are non-mechanical pumps.

\footnotetext{
${ }^{*}$ To whom correspondence should be addressed
} 
Due to these applications many researchers [8-14] have studied slip flows under different flow configurations.

In general, the thermophysical properties of the ambient fluid such as viscosity, thermal conductivity etc. are considered constant. But these physical properties may change with temperature, especially fluid viscosity, thermal conductivity etc. for most realistic fluids. The effects of the variable fluid properties on heat and mass transfer in laminar boundary layer flow have been discussed by many authors [15-19] in various situations. Recently, the convective slip flow of rarefied fluids over a wedge with variable fluid properties was studied by Rahmann and Eltayeb [20].

In the above mentioned studies the radiation effect is ignored. Many processes in engineering areas occur at high temperature and knowledge of radiation heat transfer becomes very important. The free convection interaction with thermal radiation was examined by Makinde [21]. Ibrahim et al. [22] discussed the mixed convection flow of a micropolar fluid in the presence of thermal radiation and viscous dissipation. The influence of thermal radiation on an MHD mixed convective flow was studied by Das [23]. Singh and Kumar [24] discussed boundary layer stagnation point flow of an MHD micropolar fluid towards a stretching / shrinking surface. Das et al. [25] investigated the unsteady flow of a nanofluid in the presence of thermal radiation. The impact of variable fluid properties and thermal radiation on an MHD slip flow over a flat plate was investigated by Jana and Das [26].

In the present study, the work of Rahman and Eltayeb [20] has been extended to investigate the effects of the magnetic field and thermal radiation on a convective slip flow of slightly rarefied fluids, having temperature dependent fluid properties, over a wedge with thermal jump.

\section{Formulation of the problem}

The steady two dimensional laminar flow of an electrically conducting incompressible fluid moving over the surface of a wedge under the influence of a transverse magnetic field $B(x)$ in the presence of thermal radiation with a uniform velocity $U(x)=a x^{m}$ where exponent $\mathrm{m}$ is a function of the wedge angle parameter $\beta$ such as $m=\frac{\beta}{2-\beta}(\geq 0)$. Let the $x$-axis be taken along the direction of the wedge and $y$-axis be normal to it. The angle of the wedge is given by $\beta \pi$. The magnetic Reynolds number of the flow is taken to be small enough so that the induced magnetic field is assumed to be negligible in comparison to the applied magnetic field. The viscosity and thermal conductivity of the fluid are assumed to be functions of temperature.

Under the foregoing assumptions, the governing boundary layer equations for the present problem can be written as $[6,20]$

$$
\begin{aligned}
& \frac{\partial u}{\partial x}+\frac{\partial v}{\partial y}=0, \\
& \rho\left(u \frac{\partial u}{\partial x}+v \frac{\partial u}{\partial y}\right)=\rho U \frac{d U}{d x}+\frac{\partial}{\partial y}\left(\mu \frac{\partial u}{\partial y}\right)-\sigma B^{2}(x)(u-U), \\
& \rho c_{p}\left(u \frac{\partial T}{\partial x}+v \frac{\partial T}{\partial y}\right)=\frac{\partial}{\partial y}\left[\left(\kappa+\frac{16 T_{\infty}^{3} \sigma^{*}}{3 k^{*}}\right) \frac{\partial T}{\partial y}\right]
\end{aligned}
$$

where $u, v$ are velocity components along the $x, y$-axis respectively, $\sigma$ is the electrical conductivity of the fluid, $T$ is the temperature of the fluid within the boundary layer, $\kappa$ is the thermal conductivity of the fluid, $c_{p}$ 
is the specific heat at constant pressure $p, \mu$ is the dynamic viscosity, $\sigma^{*}$ is the Stefan-Boltzmann constant and $k^{*}$ is the mean absorption coefficient.

For a slightly rarefied fluid flow, no slip boundary condition is no longer valid, therefore it needs to be replaced by a slip boundary condition and is given by

$$
u_{\text {slip }}=u_{g}-u_{w}=\lambda\left(\frac{2}{\sigma M}-1\right)\left(\frac{\partial u}{\partial n}\right)_{\text {wall }}+\frac{3 v}{4 T_{g}}\left(\frac{\partial T}{\partial s}\right)_{\text {wall }}
$$

where $\sigma_{M}$ is the tangential momentum accommodation coefficient, $\lambda$ is the mean free path, $u_{g}$ is the gas velocity at the wall, $u_{w}$ is the wall velocity, $\frac{\partial u}{\partial n}$ is the velocity gradient normal to the wall and $\frac{\partial T}{\partial s}$ is the temperature gradient along the wall.

In the present study, it is assumed that the surface of the wedge is impermeable. Therefore, there will be no flow normal to the surface and so

$$
v=0 .
$$

For rarefied fluid flows, a thermal jump condition will occur at the wall. Thus

$$
T_{\text {jump }}=T_{g}-T_{w}=\lambda\left(\frac{2}{\sigma T}-1\right) \frac{2 \gamma}{1+\gamma} \frac{\kappa}{\mu c_{p}}\left(\frac{\partial T}{\partial n}\right)_{\text {wall }}
$$

where $T_{g}$ is the temperature of the gas, $T_{w}$ is the temperature at the wall, $\sigma_{T}$ is the thermal accommodation coefficient, $\gamma$ is the ratio of specific heats and $\frac{\partial T}{\partial n}$ is the temperature gradient normal to the wall. Then matching with the free stream $(y \rightarrow \infty)$, we assume

$$
u=U(\mathrm{x}), \quad T=T_{\infty}
$$

where $T_{\infty}$ is the temperature of the ambient fluid.

Now the system of Eqs (2.1)-(2.3) is transformed into a dimensionless form. For this end, the following dimensionless variables are introduced

$$
\eta=y \sqrt{\frac{a(m+1)}{2 v_{\infty}}} x^{\frac{m-1}{2}}, \quad \psi(x, y)=\sqrt{\frac{2 a v_{\infty}}{(m+l)}} x^{\frac{m+1}{2}} f(\eta), \quad \theta(\eta)=\frac{T-T_{\infty}}{T_{w}-T_{\infty}}
$$

where $\psi(x, y)$ is the stream function, $v_{\infty}=\mu_{\infty} / \rho$ is the kinematic viscosity of the ambient fluid, $f$ is the nondimensional stream function and prime denotes differentiation with respect to $\eta$.

In order to predict the flow and heat transfer rates accurately, Ling and Dybbs [27] suggested a temperature dependent viscosity of the form

$$
\frac{1}{\mu}=\frac{1}{\mu_{\infty}}\left[1+\gamma^{*}\left(T-T_{\infty}\right)\right]
$$

where $\gamma^{*}$ is the thermal property of fluid and $\mu_{\infty}$ is the dynamic viscosity at ambient temperature.

Equation (2.9) can be written as 


$$
\frac{1}{\mu}=A\left(T-T_{r}\right)
$$

where $A=\gamma^{*} / \mu_{\infty}$, corresponds to liquids when $A>0$ and gases when $A<0$ and $T_{r}=T_{\infty}-1 / \gamma^{*}$.

The dimensionless temperature $\theta$ can also be written as

$$
\theta=\frac{T-T_{r}}{T_{w}-T_{\infty}}+\theta_{r}
$$

where $\theta_{r}=T_{r}-T_{\infty} /\left(T_{w}-T_{\infty}\right)=-1 / \gamma^{*}\left(T_{w}-T_{\infty}\right)$. Using Eq.(2.11), Eq.(2.10) becomes

$$
\mu=\mu_{\infty}\left(\frac{\theta_{r}}{\theta_{r}-\theta}\right) .
$$

The specific model for variable thermal conductivity is considered as ( Savvas et al. [28] and Chiam [15])

$$
\kappa=\kappa_{\infty}\left(1+\varepsilon \frac{T-T_{\infty}}{T_{w}-T_{\infty}}\right)
$$

where $\varepsilon$ is the thermal conductivity parameter. This relation can be written as

$$
\kappa=\kappa_{\infty}(1+\varepsilon \theta) .
$$

Applying Eqs (2.8), (2.12) and (2.14) into Eqs (2.2) and (2.3), one may obtain,

$$
\begin{aligned}
& f^{\prime \prime \prime}+\left(1-\frac{\theta}{\theta_{r}}\right) f f^{\prime \prime}+\frac{1}{\left(\theta_{r}-\theta\right)} f^{\prime \prime} \theta^{\prime}+\beta\left(1-\frac{\theta}{\theta_{r}}\right)\left(1-f^{\prime 2}\right)-M^{2}\left(1-\frac{\theta}{\theta_{r}}\right)\left(f^{\prime}-1\right)=0, \\
& (1+\varepsilon \theta+N r) \theta^{\prime \prime}+\varepsilon \theta^{\prime 2}+\operatorname{Pr}_{\infty} f \theta^{\prime}=0
\end{aligned}
$$

where $M=B(\mathrm{x}) \sqrt{\frac{2 \sigma}{\rho a(m+1) x^{m-1}}}$ is the magnetic field parameter, $\operatorname{Pr}_{\infty}=\frac{\mu_{\infty} c_{p}}{\kappa_{\infty}}$ is the ambient Prandtl number and $N r=\frac{16 T_{\infty}^{3} \sigma^{*}}{3 k^{*} \kappa_{\infty}}$ is the thermal radiation parameter.

The momentum Eq.(2.15) to have a similarity solution, the magnetic field parameter $M$ must be constant. Therefore, if it is assumed that the applied magnetic field $B(x)$ is proportional to $x^{(m-1) / 2}[29,30], M$ will be independent of $x$. Therefore, it is assumed that $B(x)=B_{0} x^{(m-1) / 2}$ where $B_{0}$ is a constant.

The non-dimensional boundary conditions for the present problem are

$$
\begin{aligned}
& f=0, \quad f^{\prime}=K f^{\prime \prime}, \quad \theta=1+\frac{1}{\operatorname{Pr}} \frac{2 \gamma}{1+\gamma} K \theta^{\prime}, \quad \text { for } \quad \eta=0, \\
& f^{\prime}=1, \quad \theta=0, \quad \text { as } \quad \eta \rightarrow \infty .
\end{aligned}
$$


where $K$ is the slip (rarefaction) parameter. It is reasonable to consider the Prandtl number as a variable which is defined as

$$
\operatorname{Pr}=\frac{\mu c_{p}}{\kappa}=\frac{\left(\frac{\theta_{r}}{\theta_{r}-\theta}\right) \mu_{\infty} c_{p}}{\kappa_{\infty}(1+\varepsilon \theta)}=\frac{1}{\left(1-\frac{\theta}{\theta_{r}}\right)(1+\varepsilon \theta)} \operatorname{Pr}_{\infty} .
$$

Using Eq.(2.18), the non-dimensional energy Eq.(2.16) can be written as

$$
(1+\varepsilon \theta+N r) \theta^{\prime \prime}+\varepsilon \theta^{\prime 2}+\operatorname{Pr}\left(1-\frac{\theta}{\theta_{r}}\right)(1+\varepsilon \theta) f \theta^{\prime}=0 .
$$

The quantities of main physical interest are the skin friction coefficient, $C_{f}$ and the Nusselt number $\mathrm{Nu}$ which are given respectively as

$$
C_{f}^{*}=\frac{K}{\sqrt{2-\beta}}\left[\beta\left(K^{2} f^{\prime \prime 2}(0)-1\right)+M^{2}\left(K f^{\prime \prime}(0)-1\right)-\frac{\theta_{r}}{\left(\theta_{r}-\theta(0)\right)^{2}} \theta^{\prime}(0) f^{\prime \prime}(0)\right]
$$

where $C_{f}^{*}=C_{f} \operatorname{Re}_{x}^{1 / 2}$ and

$$
\mathrm{Nu}^{*}=-\frac{K}{\sqrt{2-\beta}} \frac{1}{\operatorname{Pr}} \frac{2 \gamma}{1+\gamma}\left[\left\{\varepsilon-\frac{1+\varepsilon \theta(0)+N r}{\theta_{r}-\theta(0)}\right\} \theta^{\prime}(0)+\{1+\varepsilon \theta(0)+N r\} \theta^{\prime \prime}(0)\right]
$$

where $\mathrm{Nu}^{*}=\mathrm{Re}_{x}^{-1 / 2} \mathrm{Nu}$ and $\mathrm{Re}_{x}=\frac{a x^{m+1}}{v_{\infty}}$

\section{Method of solution}

The coupled non-linear ordinary differential Eqs (2.15) and (2.19) together with the boundary conditions (2.17) have been solved numerically by using the finite difference code that implements the 3stage Lobatto IIIa formula for a partitioned Runge-Kutta method. The computations were done by a program which uses symbolic computation software MATHEMATICA. The step size and convergence criteria are chosen to be 0.001 and $10^{-6}$, respectively. The asymptotic boundary conditions in Eq.(2.17) are approximated by using a value of 10 for $\eta \rightarrow \infty$.

To check the validity of the present code, the values of $f^{\prime}(\eta)$ and $f^{\prime \prime}(\eta)$ for the Falkner-Skan boundary layer equation have been calculated for $M=0, N r=0$ and $\beta=0$ and for different values of $\eta$ in Tab.1. From Tab.1, it has been observed that the data produced by the present code and those of Rahman and Eltayeb [20] show an excellent agreement and so justify the use of the present numerical code. 
Table 1. Comparison of the values of $f^{\prime}(\eta)$ and $f^{\prime \prime}(\eta)$ for various values of $\eta$.

\begin{tabular}{|c|c|c|c|c|}
\hline \multicolumn{3}{|c|}{ Rahman and Eltayeb [20] } & \multicolumn{2}{|c|}{ Present results } \\
\hline$\eta$ & $f^{\prime}(\eta)$ & $f^{\prime \prime}(\eta)$ & $f^{\prime}(\eta)$ & $f^{\prime \prime}(\eta)$ \\
\hline 0.0 & 0.000000000 & 0.469600079 & 0.000000 & 0.469600 \\
\hline 0.4 & 0.187605192 & 0.467254286 & 0.187600 & 0.467253 \\
\hline 0.8 & 0.371963288 & 0.451190073 & 0.371963 & 0.4511900 \\
\hline 1.2 & 0.545246535 & 0.410565393 & 0.545248 & 0.4105658 \\
\hline
\end{tabular}

\section{Numerical results and discussions}

In order to have an understanding of the mathematical model, the numerical results for velocity and temperature field have been presented graphically in Figs 1-5, whereas the skin friction coefficient and Nusselt number are tabulated in Tabs 2, 3 for several sets of values of the pertinent parameters. In the simulation the default values of the parameters are as follows: as $K=0.1, \beta=0.25, \theta_{r}=2.0, \varepsilon=0.5, M=0.3$, $N r=0.4, \operatorname{Pr}=0.71$ and $\gamma=1.4$ unless otherwise specified.

Figure 1 depicts the $x$-component of the translational velocity $f^{\prime}(\eta)$ and non-dimensional temperature field $\theta(\eta)$ for different values of the magnetic field parameter $M$. As the parameter value of $M$ increases in the presence of thermal radiation at the plate surface, the flow rate accelerates, thereby giving rise to a decrease in the momentum the boundary layer thickness. The fluid temperature is not significantly changed with the increase of the strength of magnetic field as shown in Fig.1. It can be noted that the fluid temperature is maximum near the boundary wall of the wedge and it decreases on increasing the boundary layer coordinate $\eta$ to approach the free stream value. Table 2 shows that the magnetic field parameter $M$ enhances the skin friction coefficient $C_{f}^{*}$ but the influence is not significant on the Nusselt number $\mathrm{Nu}^{*}$.

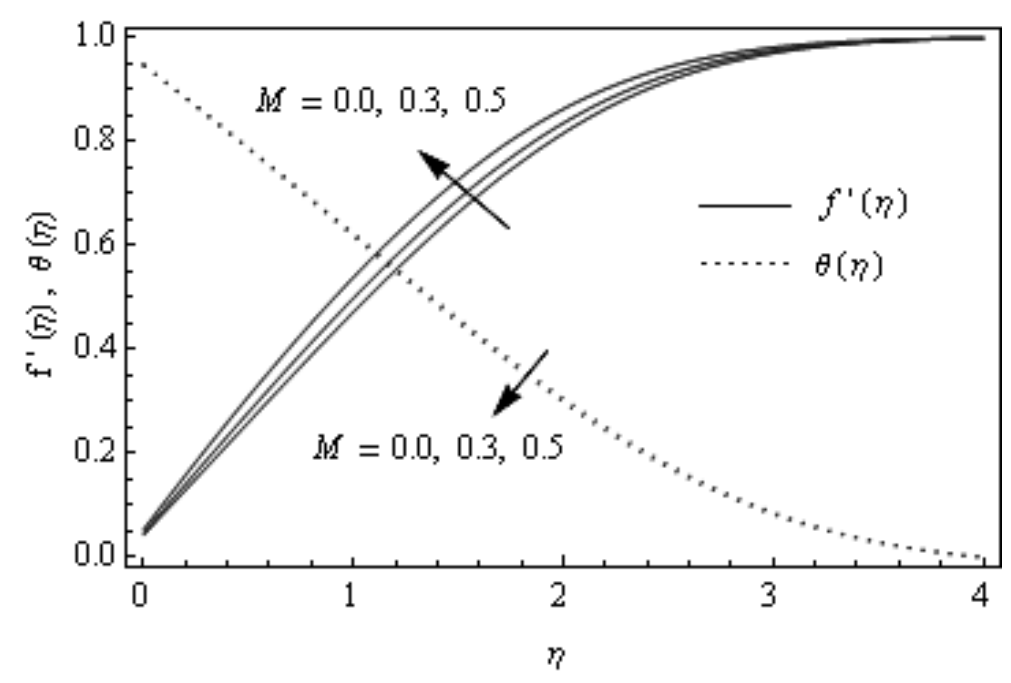

Fig.1. Magnetic effect on velocity and temperature profiles. 
Table 2. Effects of $M, K$ and $\beta$ on $C_{f}^{*}$ and $-\mathrm{Nu}^{*}$.

\begin{tabular}{|c|c|c|c|}
\hline$M$ & $K$ & $C_{f}^{*}$ & $-\mathrm{Nu}^{*}$ \\
\hline 0.0 & 0.2 & 0.0078158 & 0.0244703 \\
\hline 0.3 & & 0.0110936 & 0.0247617 \\
\hline 0.5 & & 0.0163174 & 0.0251859 \\
\hline 0.3 & 0.0 & 0.0000000 & 0.0000000 \\
\hline & 0.2 & 0.0110936 & 0.0247617 \\
\hline & 0.4 & 0.0211067 & 0.0559735 \\
\hline & 0.2 & 0.0241461 & 0.0230449 \\
\hline
\end{tabular}

The impact of the thermal radiation parameter $\mathrm{Nr}$ on the temperature profiles is presented in Fig.2. It can easily be seen that the temperature decreases as the boundary layer coordinate $\eta$ increases for a fixed value of $\mathrm{Nr}$ but the rate of the decrease is faster as the value of $\mathrm{Nr}$ goes on increasing. There is no appreciable effect of increasing the value of the radiation parameter $\mathrm{Nr}$ on the velocity profiles in the boundary layer as illustrated in Fig.2. Table 3 shows the effects of the thermal radiation parameter $\mathrm{Nr}$ on the skin friction coefficient $C_{f}^{*}$ and the Nusselt number $\mathrm{Nu}^{*}$. It is observed from this table that as $\mathrm{Nr}$ increases $\mathrm{Nu}^{*}$ increases, but the effect is opposite for $C_{f}^{*}$.

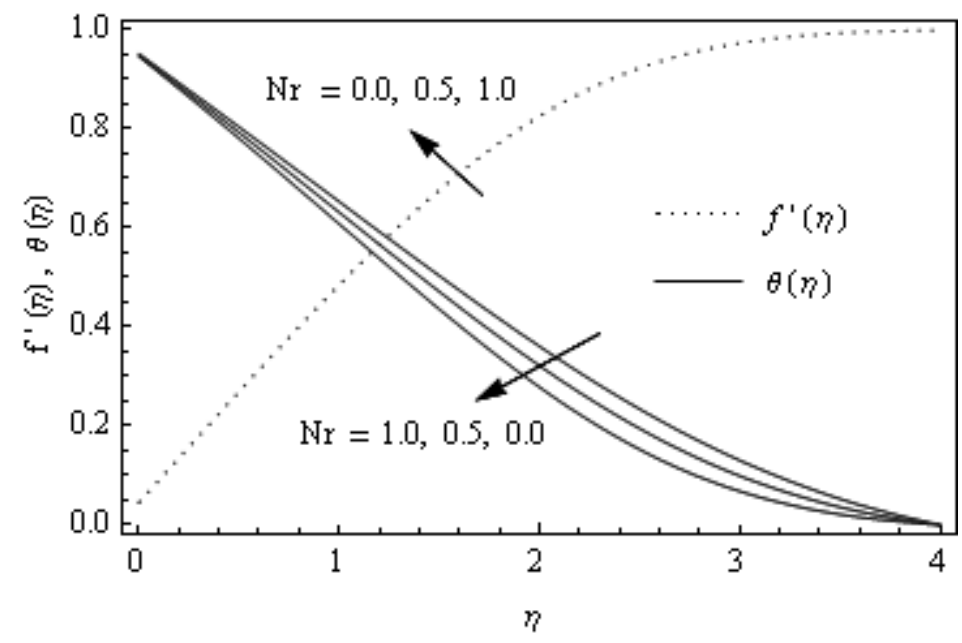

Fig.2. Thermal radiation effect on velocity and temperature profiles.

Table 3. Effects of $\theta_{r}, N r$ and $\varepsilon$ on $C_{f}^{*}$ and $-\mathrm{Nu}^{*}$.

\begin{tabular}{|c|c|c|c|c|}
\hline$\theta_{r}$ & $N r$ & $\varepsilon$ & $C_{f}^{*}$ & $-\mathrm{Nu}^{*}$ \\
\hline 1.0 & 0.3 & 1.0 & 0.4762290 & -0.0274831 \\
\hline 5.0 & & & -0.0134461 & 0.0635966 \\
\hline$\infty$ & & & -0.0213532 & 0.0913775 \\
\hline 2.0 & 0.0 & & 0.0121851 & 0.0107963 \\
\hline & 0.3 & & 0.0110936 & 0.0247617 \\
\hline & 0.6 & & 0.0102521 & 0.0370928 \\
\hline & & 0.0 & 0.0145361 & 0.0601141 \\
\hline & & 0.5 & 0.0110936 & 0.0247617 \\
\hline & & 1.0 & 0.00894979 & -0.0025097 \\
\hline
\end{tabular}


Figure 3 demonstrates the effects of the slip (rarefaction) parameter $K$ on the fluid velocity as well as the fluid temperature within the boundary layer. As the slip parameter $\mathrm{K}$ increases, the slip at the surface wall of the wedge increases. In the no-slip condition, $K$ approaches zero, so the slip velocity at the wall is equal to zero, and then $f^{\prime}(0)=0$. As the flow becomes more rarefied, friction at the surface decreases and consequently the velocity of the fluid increases. The fluid temperature decreases on increasing the rarefaction parameter $K$ in the boundary layer region as shown in Fig.3 and so the thickness of the thermal boundary layer decreases. It is evident from Tab.2 that the skin friction coefficient and Nusselt number both increase on increasing the slip parameter $K$.

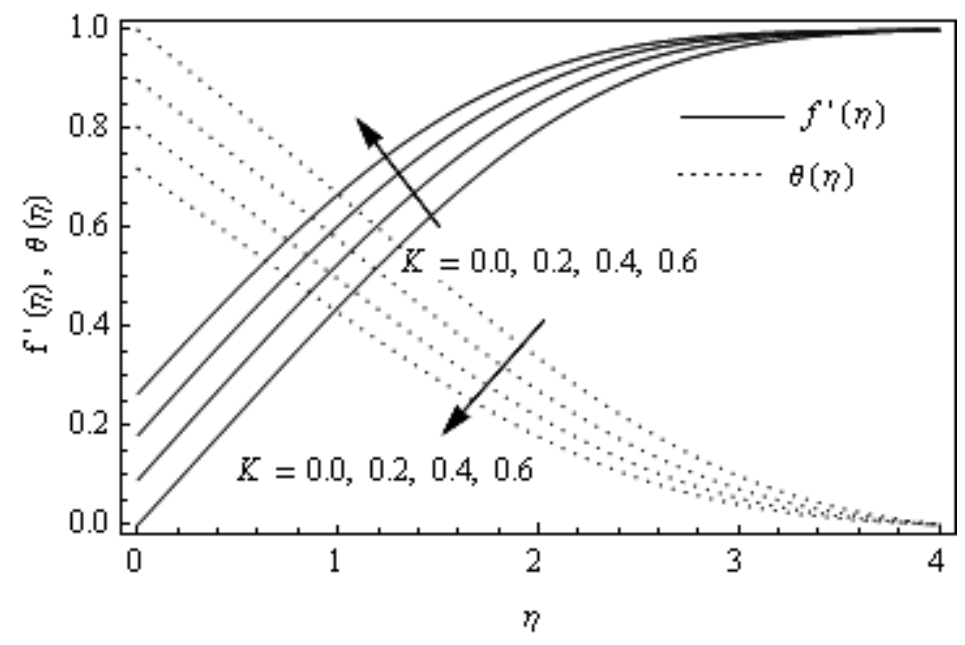

Fig.3. Slip effect on velocity and temperature profiles.

The effect of the thermal conductivity parameter $\varepsilon$ on the velocity and temperature distribution is shown in Fig.4. The effect of increasing $\varepsilon$ is to enhance the temperature and hence there would be an increase in the thermal boundary layer thickness. But there is no significant effect on fluid velocity. From Tab.3, it is observed that the thermal conductivity parameter $\varepsilon$ reduces the skin friction coefficient as well as the Nusselt number at the surface of the wedge.

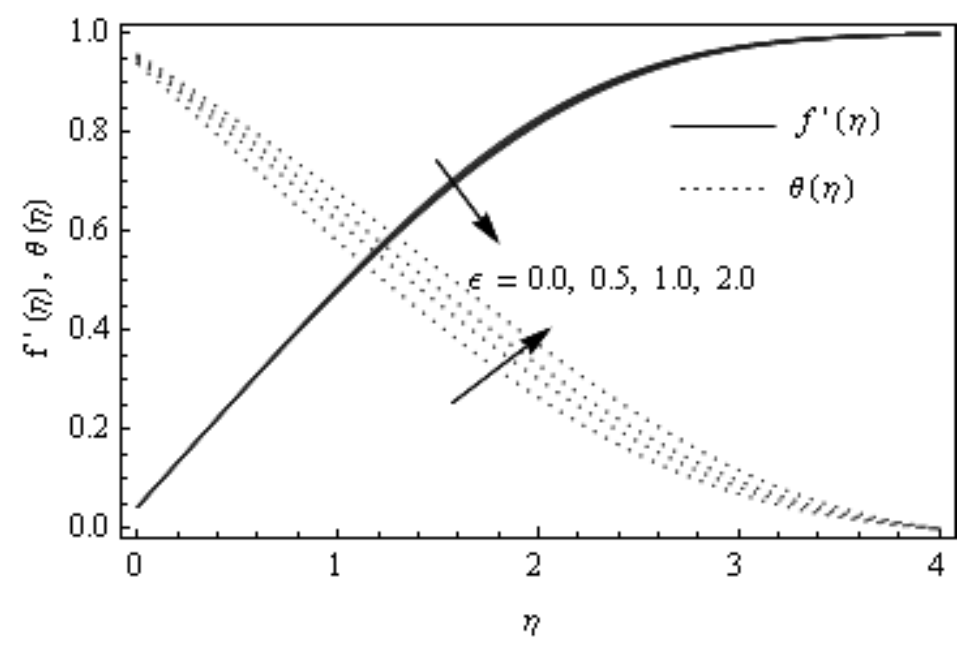

Fig.4. Effect of thermal conductivity on velocity and temperature profiles. 
The influence of the viscosity parameter $\theta_{r}$ on the velocity profile and temperature profile against the transverse coordinate $\eta$ is shown in Fig.5. The results indicate that with an increase in the parameter $\theta_{r}$, the velocity profiles increase. It should be noted that for larger values of $\theta_{\mathrm{r}}$ the influence on velocity is not prominent. It is observed from Fig. 5 that as $\theta_{r}$ increases, the thermal boundary layer thickness decreases with a consequent reduction of the temperature in the boundary layer. It is observed from Tab. 3 that $\theta_{r}$ reduces the skin friction coefficient whereas it enhances the dimensionless Nusselt number at the surface of the wedge.

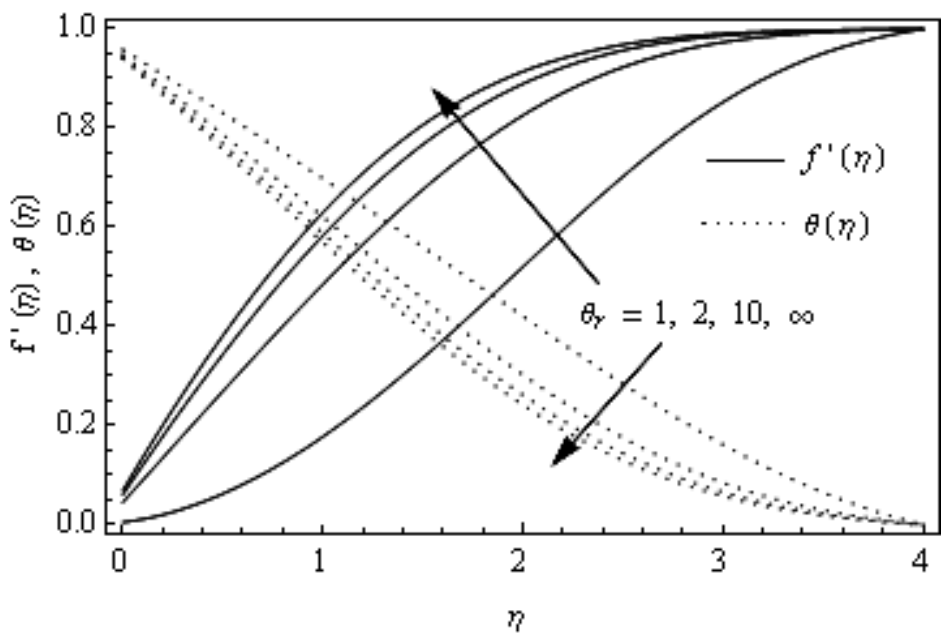

Fig.5. Effect of viscosity parameter $\theta_{r}$ on relocity and temperature profiles.

\section{Conclusions}

A mathematical model is developed to study the influence of thermal radiation and the magnetic field on a convective slip flow of electrically conducting slightly rarefied fluids, having temperature dependent fluid properties, over a wedge with a thermal jump at the surface of the boundary. Numerical results are obtained to illustrate the details of the flow and heat transfer characteristics and their dependence on material parameters. The following conclusions can be drawn from the present investigation:

(i) The skin friction coefficient decreases with an increase in the thermal radiation parameter $\mathrm{Nr}$, thermal conductivity parameter $\varepsilon$ and variable viscosity parameter $\theta_{r}$ but the effect is reverse for the magnetic field parameter $M$ and slip (rarefaction) parameter $K$.

(ii) The Nusselt number increases for the increase in the magnetic field parameter $M$, slip (rarefaction) parameter $K$, thermal radiation parameter $\mathrm{Nr}$ and variable viscosity parameter $\theta_{r}$ while it decreases for an increasing thermal conductivity parameter $\varepsilon$.

\section{Nomenclature}

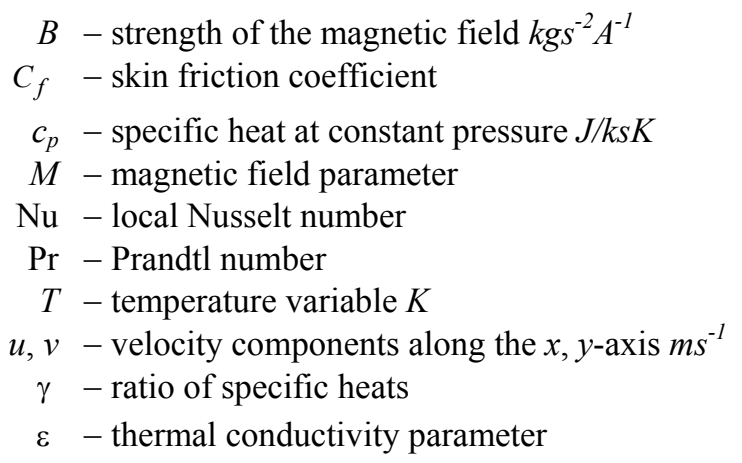


$\eta-$ similarity variable

$\theta$ - dimensionless temperature

$\mu$ - fluid viscosity $\mathrm{Ns} / \mathrm{m}^{2}$

$v \quad$ - kinematic viscosity $m^{2} s^{-1}$

$\rho-$ density of fluid $\mathrm{kgm}^{-3}$

$\sigma$ - electrical conductivity of the fluid

$\Psi$ - stream function $m^{2} s^{-1}$

Subscripts

$K$ - slip (rarefaction) parameter

$\mathrm{Nr}$ - thermal radiation parameter

$w$ - condition on the sheet

$\theta_{\mathrm{r}}$ - variable viscosity parameter

$\infty$ - condition far away from the plate

' - differentiation with respect to $\eta$

\section{References}

[1] Falkner V.M. and Skan S.W. (1931): Solutions of the boundary layer equations. - Philosophical Magazine, vol.7, pp.865-896.

[2] Rajagopal K.R., Gupta A.S. and Na T.Y. (1983): A note on the Falkner-Skan flows of a non-Newtonian fluid. International Journal of Non-Linear Mechanics, vol.18, pp.313-320.

[3] Nanousis N.D. (1999): Theoretical magnetohydrodynamic analysis of mixed convection boundary layer flow over a wedge with uniform suction or injection. - Acta Mechanica, vol.138, pp.21-30.

[4] Rashad A.M. and Bakier A.Y. (2009): MHD effects on non-Darcy forced convection boundary layer flow past a permeable wedge in a porous medium with uniform heat flux. - Nonlinear Analysis: Modelling and Control, vol.14, pp.249-261

[5] Kandasamy R., Muhaimin I., Khamis A.B. and Roslan R. (2013): Unsteady Heimenz flow of Cu-nanofluid over a porous wedge in the presence of thermal stratification due to solar energy radiation: Lie group transformation. - International Journal of Thermal Sciences, vol.65, pp.196-205.

[6] Das K. (2014): Convective slip flow of rarefied fluids over a permeable wedge plate embedded in a DarcyForchheimer porous medium. - The European Physical Journal Plus, vol.129, pp.50, pp.1-15.

[7] Gad-el-Hak M. (1999): The fluid mechanics of micro-devices-The freeman scholar lecture. - Journal of Fluids Engineering, vol.121, pp.5-33.

[8] Fang T. and Lee C.F. (2006): Exact solutions of incompressible Couette flow with porous walls for slightly rarefied gases. - Heat and Mass Transfer, vol.42, pp.255-262.

[9] Martin M.J. and Boyd I.D. (2010): Falkner-Skan flow over a wedge with slip boundary conditions. - Journal of Thermophysics and Heat Transfer, vol.24, pp.63-270.

[10] Nandeppanavar M.M., Vajravelu K., Abel M.S. and Siddalingappa M.N. (2012): Second order slip flow and heat transfer over a stretching sheet with non-linear Navier boundary condition. - International Journal of Thermal Sciences, vol.58, pp.143-150.

[11] Mukhopadhyay S. (2013) MHD boundary layer slip flow along a stretching cylinder. - Ain Shams Engineering Journal, vol.4, pp.317-324.

[12] Zheng L., Liu N., Niu J. and Zhang X. (2013): Slip and buoyancy lift effects on the mixed convection flow and radiation heat transfer of a micropolar fluid toward vertical permeable plate. - Journal of Porous Media, vol.16, pp.575-583

[13] Das K., Duari P.R. and Kundu P.K. (2014): Solar radiation effects on Cu-water nanofluid flow over a stretching 
sheet with surface slip and temperature jump. - Arabian Journal for Science and Engineering, vol.39, pp.9015- 9023.

[14] Kumar S.R. (2015): The effect of the couple stress fluid flow on MHD peristaltic motion with uniform porous medium in the presence of slip effect. - Vol.9, No.4, pp.269-278.

[15] Chiam T.C. (1998): Heat transfer in a fluid with variable thermal conductivity over a linearly stretching sheet. Acta Mechanica, vol.129, pp.63-72.

[16] Rahman M.M., Uddin M.J. and Aziz A. (2009): Effects of variable electric conductivity and non-uniform heat source (or sink) on convective micropolar fluid flow along an inclined flat plate with surface. - International Journal of Thermal Sciences, vol.48, pp.2331-2340.

[17] Prasad K.V., Vajravelu K. and Datti P.S. (2010): The effects of variable fluid properties on the hydro-magnetic flow and heat transfer over a non-linear stretching sheet. - International Journal of Thermal Sciences, vol.49, pp.603-610.

[18] Sinha A., Shit G.C. and Ranjit N.K. (2015): Peristaltic transport of MHD flow and heat transfer in an asymmetric channel: Effects of variable viscosity, velocity-slip and temperature jump. - Alexandria Engineering Journal, vol.54, pp.691-704.

[19] Malga B.S., Kishan N., Reddy V.V. and Govardhan K. (2014): Finite element analysis of fully developed free convection flow, heat and mass transfer of a MHD micropolar fluid over a vertical channel. - Vol.8, No.4, pp.219-232.

[20] Rahman M.M. and Eltayeb I.A. (2011): Convective slip flow of rarefied fluids over a wedge with thermal jump and variable transport properties. - International Journal of Thermal Sciences, vol.50, pp.468-479.

[21] Makinde O.D. (2005): Free convection flow with thermal radiation and mass transfer past a moving vertical porous plate. - International Communications in Heat and Mass Transfer, vol.32, pp.1411-1419.

[22] Ibrahim F.S., Elaiw A.M. and Bakr A.A. (2008): Influence of viscous dissipation and radiation on unsteady MHD mixed convection flow of micropolar fluids. - Applied Mathematics and Information Sciences, vol.2, pp.143-162.

[23] Das K. (2013): Effects of thermophoresis and thermal radiation on MHD mixed convective heat and mass transfer flow. - Afrika Mathematika, vol.24, pp.511-524.

[24] Singh K. and Kumar M. (2014): Melting heat transfer in boundary layer stagnation point flow of MHD micropolar fluid towards a stretching / shrinking Surface. - Vol.8, No.4, pp.403-408.

[25] Das K., Duari P.R. and Kundu P. (2014): Nanofluid flow over an unsteady stretching surface in presence of thermal radiation. - Alexandria Engineering Journal, vol.53, pp.737-745.

[26] Jana S. and Das K. (2015): Influence of variable fluid properties, thermal radiation and chemical reaction on MHD slip flow over a flat plate. - Italian Journal of Pure and Applied Mathematics, vol.34, pp.29-44.

[27] Ling J.X. and Dybbs A. (1987): Forced convection over a flat plate submersed in a porous medium: variable viscosity case. - ASME, Paper 87-WA/HT-23, ASME Winter Annual Meeting, Boston, Massachusetts, pp.13-18.

[28] Savvas T.A., Markatos N.C. and Papaspyrides C.D. (1994): On the flow of non-Newtonian polymer solutions. Applied Mathematical Modelling, vol.18, pp.14-22.

[29] Helmy K.A. (1995): MHD boundary layer equations for power law fluids with variable electric conductivity. Meccanica, vol.30, pp.187-200.

[30] Aziz A. (2009): A similarity solution for laminar thermal boundary layer over a flat plate with a convective surface boundary condition. - Communications in Nonlinear Science and Numerical Simulation, vol.14, pp.1064-1068.

Received: August 26, 2016

Revised: September 19, 2017 